\section{Feasibility and Clinical Usefulness of a Portable Biofeedback Device to Control Partial Limb Loading in Patients Treated Surgically for Bone Tumours}

\section{Benedetti Maria Grazia', Parisini Federica', Mariani Elisabetta ${ }^{1}$, Cavazzuti Lorenzo ${ }^{1 *}$, Leardini Alberto ${ }^{2}$ and Manfrini Marco $^{3}$}

${ }^{1}$ Physical Medicine and Rehabilitation Unit, Rizzoli Orthopaedic Institute, Bologna, Italy

${ }^{2}$ Movement Analysis Laboratory, Rizzoli Orthopaedic Institute, Bologna, Italy

${ }^{3}$ Orthopaedic and Traumatologic Clinic for Musculoskeletal Tumors, Rizzoli Orthopaedic Institute, Bologna, Italy

\begin{abstract}
Purpose: To verify the actual Partial Weight Bearing (PWB) after Limb Salvage Surgery (LSS) for bone tumours with respect to the orthopaedic surgeon's prescription by a biofeedback device, and to evaluate if this device can help the patient adhere to the prescribed PWB.

Methods: Eight patients with primary bone tumours who underwent LSS and were prescribed PWB in post-surgery rehabilitation were included. The load was measured in a single experimental session under three different conditions:

- free walking (pre-conditioning gait phase),

- walk with PWB with the audio-feedback device (conditioning gait phase),

- walk with PWB after audio-feedback training (post-conditioning gait phase).

Results: During the pre-conditioning gait phase, seven patients exceeded the PWB by a mean of $42.2 \%$. During the conditioning gait phase seven patients walked below the PWB threshold and one continued to exceed the prescribed PWB. During the post-conditioning gait phase, 4 patients continued to follow the prescribed PWB and 4 patients continue to exceed the PWB

*Corresponding author: Cavazzuti Lorenzo, Physical Medicine and Rehabilitation Unit, Rizzoli Orthopaedic Institute, Bologna, Italy, Tel: +39 0516366236; Fax: +39 051332396; E-mail: Iorenzo.cavazzuti@ior.it

Citation: Benedetti MG, Parisini F, Mariani E, Cavazzuti L, Leardini A, et al. (2015) Feasibility and Clinical Usefulness of a Portable Biofeedback Device to Control Partial Limb Loading in Patients treated Surgically for Bone Tumours. J Orthop Res Physiother 1: 002.
\end{abstract}

Received: December, 15 2014; Accepted: February, 12 2015; Published: February, 262015 threshold, but to a lesser extent (25\%) than during the free gait trial $(53.7 \%)$. All the patients accepted the use of the device well.

Conclusion: Patients rarely observe PWB. The ability to control the correct prescribed PWB, once perceived by the device, is generally reproduced also in the short term without the audio-feedback. The use of an audio-feedback device is suggested to improve the patient's adherence to the prescription.

Keywords: Baropodometric device; Bone tumour surgery; Partial weight bearing; Rehabilitation; Walking

\section{Introduction}

Limb Salvage Surgery (LSS) using different skeletal reconstructions (i.e., allografts, special prostheses, and composite techniques) has become firmly established over time in the treatment of bone tumours due to biomedical innovations regarding materials and methods for the reconstruction of resected bone segments, and the introduction of multi-agent chemotherapeutic protocols, that reduce edema and neoplastic mass in the period before surgery $[1,2]$. The most recent polychemotherapeutic treatments not only allow limb salvage, but also improve patients' survival and prognosis.

Among the known collateral effects that may appear after chemotherapy, particularly in young patients, growth retardation and bone mass reduction, including osteopenia and osteoporosis, are gaining more and more attention [3-10]. Patient's age at the time of treatment, compromised nutritional intake [11], reduced physical activity levels [12] and also late onset of puberty $[13,14]$ are involved in this process. Limb salvage techniques for bone tumours require a very careful progression of weight bearing on the treated limb after surgery to ensure implant stability and bone growth. The rehabilitation program usually consists of a compromise between an early stimulation of the living bone and a risky overloading which might cause complications such as fractures. A period of Partial Weight Bearing (PWB) is usually prescribed during the post-surgical period while chemotherapy cycles are administered both to allow the implant fixation in time and bone remodelling, to ensure a level of physical activity sufficient to stimulate osteoblast and thus prevent osteoporosis. This effect is crucial both in prosthetic replacements (to drive the bone anchorage to press-fit prosthetic stems) and in biological reconstructions (to facilitate the osteotomy fusion in massive allografts reconstructions or the hypertrophy of vascularised bone grafts) [15].

However the definition of partial weight bearing (also called "toe-touch weight bearing") is broad and Rubin et al., [16] showed that only $24 \%$ of surgeons were able to provide the toe-touch weight bearing definition. This uncertainty often creates doubts in patients and they have no way to check if their motor pattern is correct in observing PWB. Patients are usually taught how to walk with PWB by physical therapists by verbal instructions along with use of bathroom scales $[16,17]$. Previous studies have shown that patients are not able to walk within the limits of PWB without immediate feedback on actual loading and underloading or overloading often occurs [18-21].

Portable biofeedback devices have been developed to quantify and control objectively the actual load applied on the affected limb. Technical validation studies confirmed the good reliability of these 
systems and reproducibility of measurements [22-24]. Furthermore, several previous studies were conducted to describe and study the effectiveness of portable biofeedback devices in patients after orthopaedic surgery [18], fracture fixation [25], hip arthroplasty $[26,27]$, knee surgery [28], amputation [29,30], hip arthroplasty [31], stroke [32], lower limb fracture [33,34], and patients referred for PWB gait rehabilitation $[35,36]$, and have shown that PWB is generally not observed without biofeedback, particularly for low loading thresholds. However only one previous study [27] reported data on the effects of the biofeedback training for partial weight bearing loading at the removal of the device, and no studies were addressed to patients with musculoskeletal tumours, often young and with wide muscle removal.

The purpose of this pilot case study was to verify the actual partial weight bearing with respect to the surgeon's prescription in patients treated by limb salvage surgery for lower limb bone tumours, by a biofeedback device, and to evaluate if this device is useful to educate the patient to observe the prescribed partial weight bearing. Feasibility problems encountered during the use of this assistive technology are discussed to understand how the device can be used to its best in clinical practice.

\section{Materials and Methods}

\section{Subjects}

This feasibility study was performed on 8 consecutive patients (6 female, 2 male, mean age 32 years, range 9-62) referring to the rehabilitation outpatient clinic of Rizzoli Orthopaedic Institute. All patients underwent LSS ( 2 allografts, 5 prosthesis, 1 pubic-femoral reconstruction) in seven cases for osteosarcoma (one patient at the pelvis, two in the distal femur, four at the proximal tibia), and in one case for chondrosarcoma in the proximal femur. Walking recovery with $\mathrm{PWB}$ was prescribed to them by the orthopaedist (Table 1) during the surgical follow up. Inclusion criteria were: ability to walk with crutches for more than $20 \mathrm{~m}$, no musculoskeletal problems in other districts, no comorbilities, no cognitive impairment. At the time of the pilot study all patients were under chemotherapy treatment. However all of them were in a period of recovery from collateral effects after a cycle of chemotherapy, and no symptoms (fatigue, fever, nausea, sedation from medicaments etc.) were present. No patient presented peripheral neuropathy due to chemotherapy. A rehabilitation program for gait recovery with the prescribed partial weight bearing was ongoing at the time of the enrolment.

\begin{tabular}{|c|c|l|l|c|c|c|}
\hline Patient & $\begin{array}{l}\text { Age } \\
\text { (yrs) }\end{array}$ & $\begin{array}{l}\text { Follow-up } \\
\text { from } \\
\text { surgery } \\
\text { (days) }\end{array}$ & $\begin{array}{l}\text { Body } \\
\text { Weight } \\
\text { (Kg) }\end{array}$ & $\begin{array}{l}\text { Prescribed } \\
\text { PWB (Kg) }\end{array}$ & $\begin{array}{l}\text { Prescribed } \\
\text { PWB } \\
\text { (\% BW) }\end{array}$ & $\begin{array}{l}\text { Device } \\
\text { Thresholds } \\
\text { (Kg) }\end{array}$ \\
\hline 1 & 9 & 124 & 32 & 10 & $31 \%$ & $05-10$ \\
\hline 2 & 21 & 40 & 86 & 50 & $59 \%$ & $45-50$ \\
\hline 3 & 17 & 65 & 48 & 25 & $52 \%$ & $20-25$ \\
\hline 4 & 39 & 70 & 72 & 15 & $20 \%$ & $10-15$ \\
\hline 5 & 62 & 193 & 61 & 20 & $30 \%$ & $15-20$ \\
\hline 6 & 33 & 82 & 90 & 10 & $11 \%$ & $05-10$ \\
\hline 7 & 23 & 239 & 61 & 15 & $25 \%$ & $10-15$ \\
\hline 8 & 52 & 63 & 57 & 15 & $26 \%$ & $10-15$ \\
\hline
\end{tabular}

\section{Instrumentation}

Table 1: General Information.

The commercial biofeedback device was the Pedar mobile system (Novel $_{\mathrm{GmbH}}$, Munich, Germany), which uses flexible and size-adaptable insoles to measure plantar pressure inside the shoes. Each insole contains 99 capacitive sensors.

During data collection, the patient wears a belt containing the device box, the battery and a start/stop button. The insoles are connected to this box with cables fastened to the legs with Velcro straps.

The software of the device is designed for long-term mobile measurements without direct connection to a computer. The computer is needed to configure the internal memory device and download any data which was stored inside the flash memory of the device during data collection. This software allows two threshold load levels to be defined, usually one upper and the other lower. This is possible in only one of the two feet. The device can also provide two different audio-feedback tones when insole sensors detect loads in excess of those set as thresholds.

The measurements start with a zero deduction, which sets all insole sensors to a zero value. After this, the patient pushes the start/stop button and the measure begins. The same button is used at the end of the procedure to stop data collection.

\section{Study protocol}

The study included the quantitative instrumental evaluation through the Pedar device as the main outcome, and a qualitative assessment on patient satisfaction regarding use of the device as the secondary outcome. The Medical Ethical Committee of the institution approved the study protocol. All participants (or their curators) gave their written informed consent to take part in this feasibility study. Then they placed the Pedar insole device correctly inside their own comfortable shoes, and attached the battery, and the necessary cables (Figure 1).

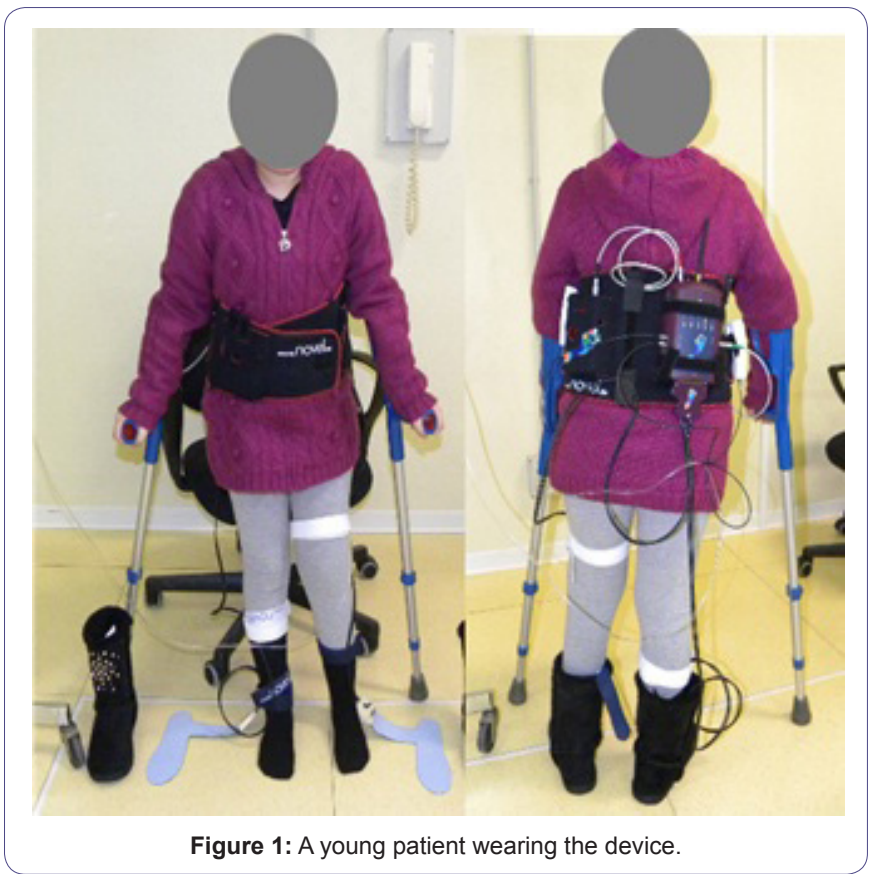

Four phases followed:

- Pre-conditioning Gait Phase: a walk of at least twenty steps on a 7-meter-long corridor during which the patient repeated the walking pattern that had been learned during the PWB training period with physiotherapist. 
- Weight definition Phase: how the device worked was explained to the patient as follows. The first audio-feedback would have been heard when the load established as the lower threshold was exceeded and a possible second audio-feedback would have been heard when the load established as the upper threshold was also exceeded. Obviously the second audio-feedback means that the patient was not observing the prescribed PWB. Therefore, it was desirable to hear only the first sound.

- Conditioning Gait Phase: a walk of at least twenty steps during which the patient received audio-feedbacks to help observe the PWB.

- Post-conditioning Gait Phase: a walk of at least twenty steps without audio-feedback in which the patient repeated the walking pattern that had been learned during the previous phase.

As a secondary outcome a non-structured satisfaction questionnaire with the following open questions was administered to the patient to investigate the level of satisfaction and usefulness of the device in their opinion:

- Did you appreciate the experimental session with the biofeedback device?

- Did you tolerate the device well?

- Do you think that the use of the device is helpful? Explain why.

\section{Data analysis}

Based upon recommended procedures [18] data from the initial and the last step were discarded. Furthermore, to carry out a more detailed analysis and the comparison of data among different conditions, data were exported to an excel file. The maximum load value for every step was hence identified, and the mean of all values was calculated for each condition (i.e., postconditioning gait, conditioning gait, post-conditioning gait) to check: significantly different from that expected. Statistically significant values were considered for $\mathrm{p}<0.05$. All the statistical analyses were performed by SPSS (SPSS Inc.).

\section{Results}

The mean number of steps was $34.2 \pm 9.6,38.2 \pm 11.8$ and $30.6 \pm 11.4$ respectively for the three gait trials with device.

During the pre-conditioning gait phase, 7 patients walked with a higher load on the treated limb with respect to the prescribed PWB. One patient did not reach the prescribed PWB (mean value -9.6\%) (Table 2).

During the conditioning gait phase, 7 patients were able to walk with a load below the maximum threshold; one patient continued to overload the treated limb, but with a reduced maximum mean value with respect to that of the pre-conditioning gait phase $(28 \%$ instead of $43.3 \%)$. During the post-conditioning gait phase 4 patients continued to remain under the prescribed PWB while the other 4 patients returned to overload the treated limb, but to a lesser extent.

Figure 2, graphically summarizes the loading values in the three conditions with respect to the PWB. In general, the value of limb loading in the three walking conditions changed on average from $23.5 \%$ in the pre-conditioning gait phase, to $-13.9 \%$ during the conditioning gait phase with the device, to $7.9 \%$ in the post-conditioning gait phase, without the device.

The paired samples t-test revealed statistically significant differences between the prescribed PWB and the load value registered during the pre-conditioning gait phase $(\mathrm{p}=0.025)$, the load value during the pre-conditioning gait phase and the load value during the conditioning gait phase $(\mathrm{p}=0.008)$ as well as the load value

\begin{tabular}{|c|c|c|c|c|c|c|c|c|c|c|}
\hline \multirow[b]{2}{*}{ Patients } & \multirow[b]{2}{*}{ Prescribed PWB $(\mathrm{Kg})$} & \multicolumn{3}{|c|}{$\begin{array}{c}\text { Pre-conditioning Gait } \\
\text { (number of steps } 34.2 \pm 9.6 \text { ) }\end{array}$} & \multicolumn{3}{|c|}{$\begin{array}{c}\text { Conditioning gait } \\
\text { (number of steps } 38.2 \pm 11.8 \text { ) }\end{array}$} & \multicolumn{3}{|c|}{$\begin{array}{c}\text { Post conditioning gait } \\
\text { (number of steps } 30.6 \pm 11.4 \text { ) }\end{array}$} \\
\hline & & Mean $(\mathrm{Kg})$ & SD & Difference \% & Mean $(\mathrm{Kg})$ & SD & Difference \% & Mean $(\mathrm{Kg})$ & SD & Difference \% \\
\hline 1 & 10 & 14.4 & 6.4 & 44.0 & 7.7 & 2.0 & -23.0 & 8.3 & 2.5 & -17.0 \\
\hline 2 & 50 & 45.2 & 5.5 & -9.6 & 46.6 & 5.9 & -6.8 & 45.8 & 10.4 & -8.4 \\
\hline 3 & 25 & 40.3 & 5.9 & 61.2 & 23.8 & 2.5 & -4.8 & 32.2 & 6.1 & 28.8 \\
\hline 4 & 15 & 24.9 & 3.7 & 66.0 & 11.1 & 2.6 & -26.0 & 13.6 & 3.2 & -9.3 \\
\hline 5 & 20 & 21.3 & 3.2 & 6.5 & 17.1 & 5.0 & -14.5 & 19.6 & 2.3 & -2.0 \\
\hline 6 & 10 & 17.0 & 2.2 & 70.0 & 6.6 & 1.7 & -34.0 & 11.4 & 1.9 & 14.0 \\
\hline 7 & 15 & 23.4 & 5.2 & 56.0 & 13.5 & 2.7 & -10.0 & 17.2 & 3.2 & 14.7 \\
\hline 8 & 15 & 21.5 & 5.9 & 43.3 & 19.2 & 4.0 & 28.0 & 21.4 & 6.0 & 42.7 \\
\hline
\end{tabular}

Table 2: Data on actual loading in the three gait conditions with respect to prescribed PWB.

- The correspondence between the prescribed PWB and the real load put on the affected limb during free walking.

- If conditioning with Pedar device enables patients to learn and put into practice the senso-motorial pattern required for walking with PWB.

- If the ability to control the limb loading acquired when using the device is maintained in the short term without audio-feedback.

As for the statistical analysis the paired samples t-test was used to analyse differences among mean values of prescribed PWB, free walking load, walking load with device, and walking load without device. A binomial test was also carried out to check if the proportion of cases having a $25^{\text {th }}$ percentile under and a $75^{\text {th }}$ percentile above an ideal threshold (almost 20\% of prescribed PWB) was registered during the conditioning gait phase and the postconditioning gait phase $(\mathrm{p}=0.019)$. No difference was found between the load value during the PWB and the post-conditioning gait phase (Table 3).

Finally, the binomial test, performed to check if the proportion of cases that had a $25^{\text {th }}$ percentile under and a $75^{\text {th }}$ percentile above an ideal threshold of $\pm 3 \mathrm{Kg}$ (almost $20 \%$ of prescribed PWB) showed that the mean load value was not significantly below the values of $-3 \mathrm{Kg}$ in any of the three trials. Only the mean load value during the pre-conditioning gait phase was significantly above the ideal threshold of $+3 \mathrm{Kg}(\mathrm{p}=0.004)$ (Figure 3$)$.

Concerning the satisfaction of the patients with respect to the use of the device, five patients highly appreciated the experimental session with the device, and three patients said it was useful. 


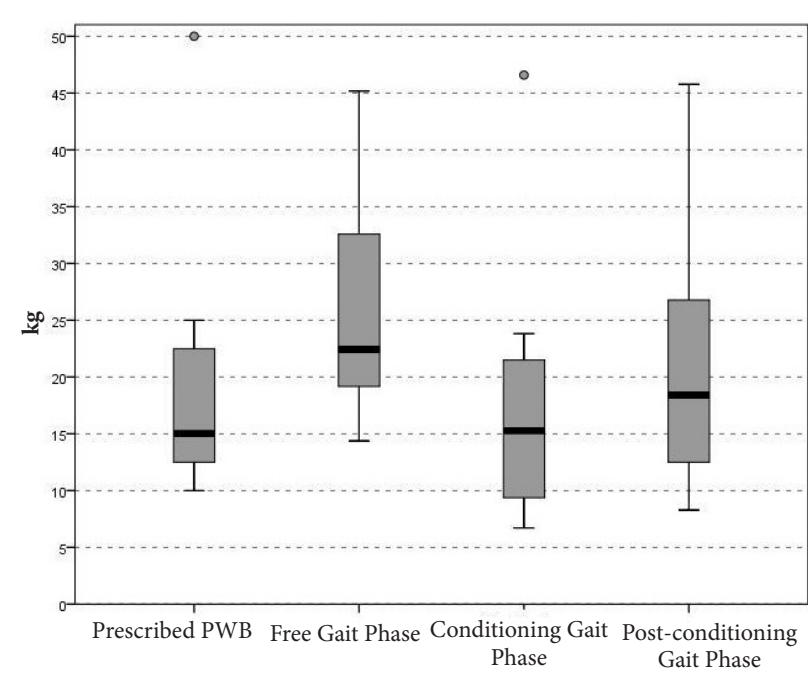

Figure 2: Median values and confidence intervals of PWB in $\mathrm{Kg}$ in each gait condition, examined with paired t-test.

\begin{tabular}{|l|c|c|c|c|c|c|}
\hline & $\begin{array}{l}\text { Difference } \\
\text { (Kg) }\end{array}$ & SD & SE & \multicolumn{2}{|l|}{$\begin{array}{l}\text { Confidence } \\
\text { interval } \\
\mathbf{9 5 \%}\end{array}$} & p \\
\hline $\begin{array}{l}\text { Pre-conditioning Gait Phase } \\
\text { vs prescribed PWB }\end{array}$ & 6.0 & 6.0 & 2.1 & -0.99 & 11.0 & 0.025 \\
\hline $\begin{array}{l}\text { Pre-conditioning Gait Phase } \\
\text { vs Conditioning Gait Phase }\end{array}$ & 7.8 & 6.0 & 2.1 & 2.8 & 12.8 & 0.008 \\
\hline $\begin{array}{l}\text { Conditioning Gait Phase vs } \\
\text { Post-conditioning Gait } \\
\text { Phase }\end{array}$ & -3.0 & 2.8 & 1.0 & -5.3 & -0.7 & 0.019 \\
\hline $\begin{array}{l}\text { Post-conditioning Gait } \\
\text { Phase vs prescribed PWB }\end{array}$ & 1.2 & 3.9 & 1.4 & -2.1 & 4.5 & 0.43 \\
\hline
\end{tabular}

Table 3: Differences among the three gait conditions.

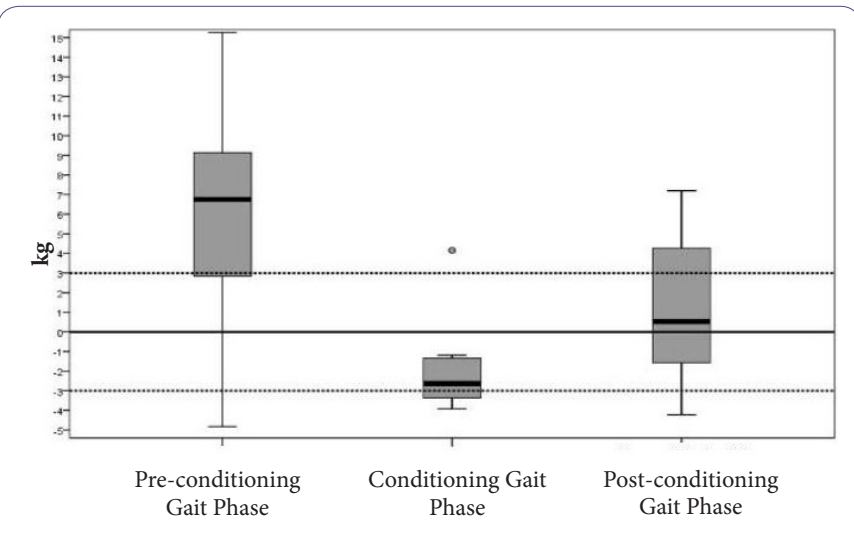

Figure 3: Median values differences (delta) and confidence intervals of PWB to check if the proportion of cases that had a $25^{\text {th }}$ percentile under and a $75^{\text {th }}$ percentile over an ideal threshold of $\pm 3 \mathrm{Kg}$ (almost $20 \%$ of prescribe PWB) by the Binomial Test. Only the mean loading value during the Free Gait Phase was significantly $(p=0.004)$ over the ideal threshold of $+3 \mathrm{Kg}$.

With respect to the device used during the experimental session, two patients stated that the waist belt of the device was too heavy and one of them (the youngest one) also reported that the belt size was too large. One patient said that the two tones of audio-feedback should be more differentiated. Three patients said that the device should be worn at home, and one patient added that the device should be worn during the rehabilitation sessions. Seven patients stated that the use of this device was helpful, because it allowed them to understand the actual load applied on the affected limb thanks to audio-feedback. Only one patient said it is not very useful if its use is not continuous, because having finished the test, the patient finds himself once again without device and unable to know how much load is put on the affected limb.

\section{Discussion}

The study provided evidence of the feasibility in using a portable bio-feedback device for partial weight bearing control in patients undergoing primary bone tumour reconstructive surgery during a rehabilitation session. Analyzing the load values recorded during the pre-conditioning gait phase, when the patient tried to repeat the walking pattern learned during the PWB training period with the therapist, it was evident that the prescribed PWB was generally not observed. The majority of patients exceeded, on average, the prescribed load. Only one patient did not, as she was very frightened to put load on the treated limb. These findings are in agreement with those of the literature [18,25-36].

Moreover, conditioning with the biofeedback device to walk according to the prescribed PWB allowed greater adherence to the prescribed amount of load. Only one patient continued to exceed the prescribed PWB while using the device, whereas the other seven patients on average remained below the prescribed load (represented by the upper threshold). Concerning the load values recorded during the post-conditioning gait phase without the device, performed shortly after the phase with the device, it can be said that in the short term there was a satisfactory adherence to the prescribed PWB. As a limit of the present study however, beside the small sample of patients, the lack of a follow up control of the effects of the conditioning training is to be taken into consideration. To be able to confirm the behaviour of patients over time with respect to the conditioning received, the period of follow-up should be extended. In fact Pataky et al., [27] reported that conditioning with biofeedback could not be effective in the long term. Furthermore, the authors agree with Hurkmans et al., [26] on the importance of measuring not only the amount of load but also the duration (amount of steps) of load. As repetitive impulses are typical of walking, complications may in fact occur even as a result of repetitive stresses in long-term weight bearing above the threshold. Future studies are recommended on the long term adherence to the prescribed partial weight bearing loading after conditioning patients with a rehabilitation training through biofeedback devices.

Patients in the present study generally positively accepted conditioning with a biofeedback device, especially the youngest ones who regarded the sound signal as a game. Actually, the use of the device was considered to be helpful for managing patients' difficulties related to PWB instructions, and it should be introduced as soon as possible during rehabilitation training for gait recovery with PWB. Chow et al., [33] reported that among factors influencing the ability to partially bear weight, muscle power and mental state are the most significant. Other factors such as age, body weight, and type of surgery do not seem to have any effect. Muscle power is also a critical aspect for patients treated for bone tumours. Often in fact a large part of muscles around the tumours are removed thus decreasing the ability to control the treated limb. Clark et al., [37] showed that, during walking with assistive devices, muscle activation patterns varied with weight-bearing load. The leg extensor muscles appeared to incur a greater reduction in muscle activity when compared with their flexor 
counterparts. In addition, we observed that the use of the biofeedback device could be helpful also when teaching patients to use forearm crutches or other devices correctly, as many patients adopt an unfavourable technique when using the crutches by loading them too late or unloading them too early [28].

This issue can justify future studies on relationships between residual muscle function after tumour removal and ability to control weight bearing on the treated limb. Moreover satisfaction of patients in the use of biofeedback devices should be better explored my means of a validated questionnaire.

Tools for monitoring the adequate load on the treated limb are expected to be very useful in this clinical context. Although introduced many years ago, surprisingly the use of these tools is not yet widespread in clinical practice. Unfortunately, at the moment, these devices are still very expensive, and their use is confined to the laboratory setting. However, even with such expensive devices, the biofeedback training seems to provide benefit to patients in terms of more clear awareness of their performance according to the medical prescription, relevant for a safe outcome of limb salvage surgery.

Patients and physiotherapists participating in the present study emphasized the usefulness of having a similar device in the gym or even at home, when patients walk unsupervised. A rapid evolution towards cheaper and more pervasive assistive technology is desirable in the near future.

\section{References}

1. Capanna R, van Horn JR, Biagini R, Ruggieri P, Bettelli G, et al. (1986) Reconstruction after resection of the distal fibula for bone tumor. Acta Orthop Scand 57: 290-294.

2. Eckardt JJ, Eilber FR, Rosen G, Mirra JM, Dorey FJ, et al. (1991) Endoprosthetic replacement for stage IIB osteosarcoma. Clin Orthop Relat Res 270 : 202-213.

3. Henderson RC, Madsen CD, Davis C, Gold SH (1998) Longitudinal evaluation of bone mineral density in children receiving chemotherapy. J Pediatr Hematol Oncol 20: 322-326.

4. Holzer G, Krepler P, Koschat MA, Grampp S, Dominkus M, et al. (2003) Bone mineral density in long-term survivors of highly malignant osteosarcoma. $J$ Bone Joint Surg Br 85: 231-237.

5. Kaste SC, Ahn H, Liu T, Liu W, Krasin MJ, et al. (2008) Bone mineral density deficits in pediatric patients treated for sarcoma. Pediatr Blood Cancer 50: 1032-1038.

6. Muller C, Winter CC, Rosenbaum D, Boos J, Gosheger G, et al. (2010) Early decrements in bone density after completion of neoadjuvant chemotherapy in pediatric bone sarcoma patients. Musculoskeletal Disorders 11: 287.

7. Pfeilschifter J, Diel IJ (2000) Osteoporosis due to cancer treatment: pathogenesis and management. J Clin Oncol 18: 1570-1593.

8. Ruza E, Sierrasesumaga L, Azcona C, Patino-Garcia A (2006) Bone minera density and bone metabolism in children treated for bone sarcomas. Pediatr Res 59: 866-871.

9. Van Leeuwen BL, Kamps WA, Jansen HW, Hoekstra HJ (2000) The effect of chemotherapy on the growing skeleton. Cancer Treat Rev 26: 363-376.

10. Wasilewski-Masker K, Kaste SC, Hudson MM, Esiashvili N, Mattano LA, et al. (2008) Bone mineral density deficits in survivors of childhood cancer: long-term follow-up guidelines and review of the literature. Pediatrics 121 705-713.

11. Sala A, Pencharz P, Barr RD (2004) Children, cancer, and nutrition - A dy namic triangle in review. Cancer 100: 677-687.
12. Armstrong GT, Chow EJ, Sklar CA (2009) Alterations in Pubertal Timing following Therapy for Childhood Malignancies. Endocr Dev 15: 25-39.

13. Janz KF, Medema-Johnson HC, Letuchy EM, Burns TL, Gilmore JM, et al. (2008) Subjective and objective measures of physical activity in relationship to bone mineral content during late childhood: the lowa Bone Development Study. Br J Sports Med 42: 658-663.

14. Janz KF, Gilmore JM, Burns TL, Levy SM, Torner JC, et al. (2006) Physical activity augments bone mineral accrual in young children: The lowa Bone Development study. J Pediatr 148: 793-799.

15. Taddei F, Viceconti M, Manfrini M, Toni A (2003) Mechanical strength of a femoral reconstruction in paediatric oncology: a finite element study. Proc Inst Mech Eng H 217: 111-119.

16. Rubin G, Monder O, Zohar R, Oster A, Konra O, et al. (2010) Toe-touch weight bearing: myth or reality? Orthopedics 33: 729 .

17. Hurkmans HL, Bussmann JB, Benda E, Verhaar JA, Stam HJ (2003) Techniques for measuring weight bearing during standing and walking. Clin Biomech (Bristol Avon) 18: 576-589.

18. Hershko E, Tauber C, Carmeli E (2008) Biofeedback versus Physiotherapy in Patients with Partial Weight-Bearing. Am J Orthop (Belle Mead NJ) 37: 92-96.

19. Bohannon RW, Waters G, Cooper J (1989) Perception of unilateral lower extremity weightbearing during bilateral upright stance. Percept Mot Skills 69: $875-880$.

20. Bohannon RW, Kelly CB (1991) Accuracy of weightbearing at three target levels during bilateral upright stance in patients with neuropathic feet and control subjects. Percept Mot Skills 72: 19-24.

21. Schaefer L, Bohannon RW (1990) Perception of unilateral weightbearing during unilateral and bilateral upright stance. Percept Mot Skills 71: 123-128.

22. Hsiao H, Guan J, Weatherly M (2002) Accuracy and precision of two in-shoe pressure measurement systems. Ergonomics 45: 537-555.

23. Hurkmans HL, Bussmann JB, Selles RW, Horemans HL, Benda E, et a (2006) Validity of the Pedar Mobile system for vertical force measurement during a seven-hour period. J Biomech 39: 110-118.

24. Hurkmans HL, Bussmann JB, Benda E, Verhaar JA, Stam HJ (2006) Accuracy and repeatability of the Pedar Mobile system in long-term vertical force measurements. Gait Posture 23: 118-125.

25. Vasarhelyi A, Baumert T, Fritsch C, Hopfenmüller W, Gradl G, et al (2006) Partial weight bearing after surgery for fractures of the lower extremity- is it achievable? Gait Posture 23: 99-105.

26. Hurkmans HL, Bussmann JB, Selles RW, Benda E, Stam HJ, et al (2007) The difference between actual and prescribed weight bearing of total hip patients with trochanteric osteotomy: long-term vertical force measurements inside and outside the hospital. Arch Phys Med Rehabil 88: 200-206.

27. Pataky Z, De Leon Rodriguez D, Golay A, Assal M, Assal JP, et al (2009) Biofeedback training for partial weight bearing in patients after total hip arthroplasty. Arch Phys Med Rehabil 90: 1435-1438.

28. Krause D, Wiinnemann M, Erlmann A, Hölzchen T, Mull M, et al (2007) Biodynamic feedback training to assure learning partial load bearing on forearm crutches. Arch Phys Med Rehabil 88: 901-906.

29. Flowers WC, Cullen CP, Tyra KP (1986) A preliminary report on the use of practical biofeedback device for gait training of above-knee amputees. J Rehabil Res Dev 23: 7-18.

30. Chow DH, Cheng CT (2000) Quantitative analysis of the effects of audio biofeedback on weight-bearing characteristics of persons with transtibial amputation during early prosthetic ambulation. J Rehabil Res Dev 37: 255-260.

31. Tveit M, Kärrholm J (2001) Low effectiveness of prescribed partial weight bearing. Continuous recording of vertical loads using a new pressure-sensitive insole. J Rehabil Med 33: 42-46. 
32. Batavia M, Gianutsos JG, Kambouris M (1997) An Augmented auditory feedback device. Arch Phys Med Rehabil 78: 1389-1392.

33. Chow SP, Cheng CL, Hui PW, Pun WK, Ng C (1992) Partial weight bearing after operations for hip fractures in elderly patients. J R Coll Surg Edinb 37: 261-262.

34. North K, Maass SD, Hitchcock RW (2010) An insole sensor for recording weight bearing behavior during tibial fracture rehabilitation. Conf Proc IEEE Eng Med Biol Soc 2010: 1856-1859.
35. Kaplan Y (2007) The use of a new biofeedback insole weight-bearing measuring device in the assessment and rehabilitation of soccer players: A case study review. J Sports Science Med 10: 30-43.

36. Isakov E (2007) Gait rehabilitation: a new biofeedback device for monitoring and enhancing weight-bearing over the affected lower limb. Eura Medicophys 43: 21-26.

37. Clark BC, Manini TM, Ordway NR, Ploutz-Snyder LL (2004) Leg muscle activity during walking with assistive devices at varying levels of weight bearing. Arch Phys Med Rehabil 85: 1555-1560. 